\title{
LIMNOLOGICAL STUDIES RELATED TO PHYSICO-CHEMICAL CHARACTERISTICS OF SHRI SHARANABASAVESHWARA LAKE WATER OF GULBARGA CITY
}

\author{
Shivasharanappa ${ }^{1}$, Bettad Prakash ${ }^{2}$ \\ ${ }^{1}$ Associate Professor, ${ }^{2}$ M.Tech Student, Dept of Civil Engineering, P. D. A. College of Engineering (Autonomous \\ Institution) Gulbarga, Karnataka State, India,shivasharanappa.g@gmail.com,prakash.bettad@gmail.com
}

\begin{abstract}
The present study deals with the seasonal limnological investigation related to physico-chemical characteristics of water of the Shri Sharanabasaveshwara Lake, Karnataka State. Besides temperature and meteorological data, 11 limnological parameters were tested following standard methods at 5 sampling sites/stations of the lake. The physico-chemical parameters ( $p H, D O, B O D, H C O_{3}, T D S$, Chloride, Nitrate, $\mathrm{TH}, \mathrm{Ca}, \mathrm{Mg}$ and $\mathrm{SO}_{4}$ etc.) showed distinct variation in the lake sites.
\end{abstract}

Keywords: Limnology, Physico-chemical parameters, Shri Sharanabasaweshwara Lake

\section{INTRODUCTION}

Wetland i.e., lakes, jheels, beels etc., have a variety of linkages for energy and nutrient exchange with surrounding watersheds and air sheds. The inland freshwater ecosystem both lentic and lotic, are being increasingly subjected to greater stress form various human activities (Wood and Gibson, 1974; Hemasundaram, 2003). The eutrophication of water means enrichment with nutrients and the resulting degradation of its quality accompanied by luxuriant growth of micro and macrophytes are recognized as a major problem in the developed as well as in developing countries. The enrichment of nutrients occurs due to the disposal of domestic and farm sewage, industrial effluents and from the runoff from surrounding areas. Vollenweider (1968) pointed out that the domestic sewage is a major source of eutrophication. With the constantly increasing eutrophication of surface waters more and more attention is being paid to investigations of the bottom sediments of wetlands (Kajak, 1979; Golterman and Qude, 1991; Freeman et al., 1993; Clair et al., 1996; Dortch, 1996; Craft, 1997; Ansari and Kumar, 2003). Although considerable investigations have been made by the researchers but a little information is known about the systematic limnological studies in Gulbarga Shri. Sharanbasveshwara Lake However, it is important to note that no qualitative, quantitative or ecological study on limnology was made so far from Shri Sharanasveshwara Lake. Shri Sharanabasveshwara lake has received various types of sewage i.e., domestic sewage, cattle waste etc. Hence an attempt into the limnological investigation in relation to the different physicochemical characteristics of water of the lake was taken up in the present investigation.

\subsection{Information about Shri Sharanabasaweshwara}

Lake:

The Shri Sharanabasaweshwara Lake is situated in the heart of the city and is also known is Jagat Tank. The eastern, northern and southern boundaries are flanked by roads, while a part of western bank is linked through a narrow channel with the adjacent moat of the Gulbarga Fort which a part of the city. This tank spreads over an area of $0.275 \mathrm{sq} . \mathrm{km}$ the mean depth is 2.2 meters with a maximum being 1.2 meters during the dry period. The tank is fully utilized by the Fisherman's Cooperative society. Shri Sharanabasaweshwara Lake exists from many many decades. Since from early days this lake is used for cattle rearing, for bathing, for fishing, and also women used to wash their household cloths. After few decades, the municipal administration joined the sewage drains from Ward A (Shahabazaar area) of Gulbarga City into this lake. That's the point from which the lake got polluted \& silted up. Incidences of fish kill happened 2 to 3 times. Very recently the City administration has taken up steps to desilt the tank and added fresh water in it, apart from that they also constructed stone lining/pitching \& fencing throughout the periphery of the lake. Now it is well protected from animals and anthropogenic hindrances. It is now being used for contractual recreational boating only.

\subsection{Objectives of the study:}

The main objectives of the current study are as following.

1. Studying the Physico-chemical characteristics of lake water.

2. Knowing the suitability of water for aquatic life, recreation etc. 


\section{MATERIALS AND METHODS}

Study Area: Gulbarga City is a divisional head quarter of five revenue districts of, Bidar, Gulbarga, Yadgir, Raichur, Bellary, Koppal and is also having Gulbarga University, Central University and VTU regional office. It is situated on Mumbai - Chennai Section of the Central Railway and is at a distance of $640 \mathrm{Kms}$. from the capital of Karnataka, the Bangalore. It is sufficiently developed in the field of education commerce and industries. There are many educational institutes including Medical, Engineering, Arts, Science and Commerce Colleges. The city has all India Radio Broadcasting station and Television transmitting center. Gulbarga is also a pilgrimage place of two religious centers, viz., Shri Sharanabasaveshwara Temple and Hazrat Khaja Bande Nawaz Darga. The city is situated at latitude of $17^{\circ} 20^{\prime}$ and longitude of $76^{\circ} 50^{\prime}$. It has a general slope of North-West to South-East.. The town lies between the contours $1570 \mathrm{~m}$ and $1460 \mathrm{~m}$ and the average elevation above the mean sea level is about $458 \mathrm{~m}$. The ground level varies from $488 \mathrm{~m}$ to $420 \mathrm{~m}$. Gulbarga city falls under one of the hottest regions. The climate is usually warm during most of the year. The maximum temperature in summer is $46^{\circ} \mathrm{C}$ during March to May and minimum temperature is $10^{\circ} \mathrm{C}$ during December average temperature is $36^{\circ} \mathrm{C}$. The winds are generally light and moderate with some increase in force during the latter part of summer and monsoon. The rainy seasons occurs during June to October following by winter season from November to January. The average annual rainfall is $691.7 \mathrm{~mm}$. The mean annual maximum humidity observed in the morning is $60 \%$ and minimum in the evening is $40 \%$. Geologically the area is composed of Deccan traps of tertiary period and shale and lime stones in Bhima series. Deccan traps occupy $3 / 4$ of the area as horizontal flows of Basaltic lava. Different varieties of traps are present in the study area, (1) Hard massive trap (2) Jointed weathered trap (3) Zeolitic trap. The weathering of trap varies from place to place depending on the elevation. In a few places in between two trap flows bole beds (Inter trappings) are present, these are good aquifers. The jointed Zeolitic and weathered trap also act as good aquifers in the area. The location map of Shri Sharanabasaveshwara Lake is shown in figure1. Sampling points chosen are at North-West corner S1, North-East corner S2, Centre of lake S3, SouthWest corner S4 and South-East corner S5. The inlet is near sampling pointS1 and the outlet is near sampling point S5. The physico-chemical characteristics are analysed as per standard procedural methods. For statistical analysis viz., Correlation coefficients and Factor analysis MINITAB software is utilised.

Correlation Coefficients (r): The degree of relationship between the variables is measured through the correlation analysis. The measure of correlation is called correlation coefficient, represented by symbol ' $r$ '. The Karl Pearson's formula is

$$
r=\frac{N \sum\left(X_{i} Y_{i}\right)-\left(\sum X_{i}\right) \cdot\left(\sum Y_{i}\right)}{\sqrt{\left[N \sum X_{i}{ }^{2}-\left(\sum X_{i}\right)^{2}\right]\left[N \sum Y_{i}{ }^{2}-\left(\sum Y_{i}\right)^{2}\right]}}
$$

Factor Analysis: Factor analysis is a very powerful technique which provides information on the meaningful parameters which describe the whole data set rendering data reduction with minimum loss of information (Singh K. P. et. al,). FA is a quantification of the significance of variables that explain the observed grouping and patterns of the inherit properties of the individual objects (Kowalkowski T. et. al,). FA allows the explaining of related parameters by only one factor (Singh K. P. et. al, Kowalkowski T. et. al, Boyacioglu. H. et. al,). FA exposes the important factor responsible for variation in ground water quality and eventually leads to sources identification of ground water pollution. In this study, FA was applied to extract the most significant factors and to reduce the contribution of less significant variables to simplify even more of the data structure coming from factor analysis. The factors obtained were further subjected to varimax rotation according to well established rules to maximize differences between the variables and facilitate easy interpretation of the data (Singh K. P. et. al, Kowalkowski T. et. al, Boyacioglu. H. et. al,). The rotating axis defined by factor analysis generates varimax factor (Factor1, Factor2....) which can further reduce the contribution of variable with minor significance. The classification of the factor loading as 'strong', 'moderate' and 'week' corresponds to absolute loading of > 0.75, 0.75-0.50 and 0.50-0.30, respectively (Liu. C.W. et. al,).

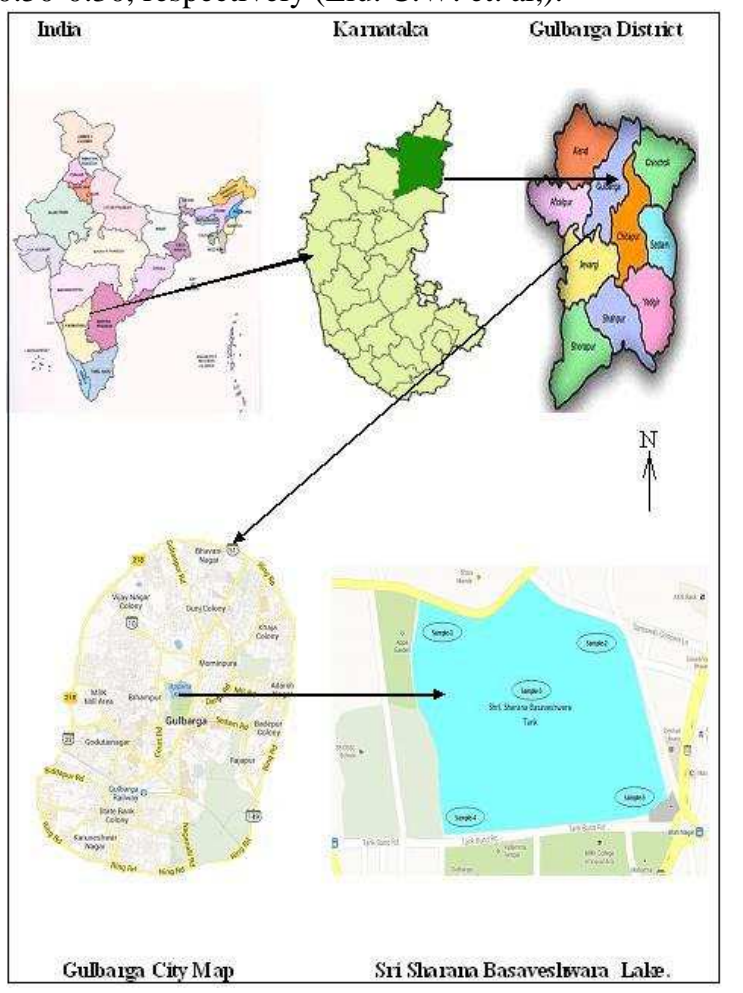

Figure1: Location Map of Shri Sharanabasaweshwara Lake 


\section{RESULTS AND DISCUSSIONS}

The physico-chemical characteristics are analysed for all the sampling points and are presented in the table 1 to table5. Correlation coefficients are worked out for individual samples and are presented in the table 6 to table 10. Factor analysis also worked out for all the individual samples and is presented in table 11 to table 15 .

Table 1: Characteristics of Lake water at sampling point 1

\begin{tabular}{|c|c|c|c|c|c|c|c|c|c|c|c|c|c|}
\hline te & atms & & pH & kalinity & DO & $B O D$ & TDS & TH & $\mathrm{Ca}$ & $\mathrm{Mg}$ & $\mathrm{NO}_{3}$ & $\mathrm{SO}_{4}$ & cl \\
\hline 5-Nov & 30.2 & 1.4 & 8.8 & 19.32 & 12 & 07.1 & 342 & .4 & 2.05 & 47.35 & 02 & 96 & 235 \\
\hline & & & & & & & & & & & & & 32.64 \\
\hline & & & & & & & & & & & 87 & 01 & 230,27 \\
\hline & & & & & & & & & & & & & \\
\hline & & & & & & & & & & & & & 25.55 \\
\hline & & & & & & & & & & & & & \\
\hline & & & & & & & & & & & & & 20.8 \\
\hline & & & & & & & & & & & & 99 & 21812 \\
\hline & & & & & & & & & & & & & $\begin{array}{l}216.05 \\
\end{array}$ \\
\hline & & & & & & & & & & & & & 213.6 \\
\hline & & & & & & & & & & & & & \\
\hline $8-D$ & & 21.3 & 8.8 & & 8. & & & & & & & & 16.16 \\
\hline $11-\mathrm{D}$ & & & & & & & & & & & & & 170 \\
\hline & & & & & & & & & & & & & \\
\hline & & & & & & & & & & & & & \\
\hline & & & & & & & & & & & & & 2.2 \\
\hline & & & & & & & & & & & & & \\
\hline & & & & & & & & & & & & & \\
\hline & 30 & & & & & & & & & & & & 35 \\
\hline M & 22 & 21. & 8. & & & & & & & & & 11 & $\sqrt{32}$ \\
\hline Vle & 26. & & & & 8. & & & & & 42 & & 78 & 62 \\
\hline 5. & & 1.0 & & & & & & & & & & & \\
\hline & & .047 & & & & .0330 & 0.014 & & & 0.007 & .06 & 0.084 & \\
\hline
\end{tabular}

pH: Hydrogen Ion Concentration, DO: Dissolved Oxygen in $\mathrm{mg} / \mathrm{L}$, BOD: Bio-chemical Oxygen Demand in $\mathrm{mg} / \mathrm{L}$, TDS: Total Dissolved Solids in mg/L, TH: Total Hardness in mg/L, $\mathrm{Ca}$ : Calcium Hardness in $\mathrm{mg} / \mathrm{L}, \mathrm{Mg}$ : Magnesium Hardness in $\mathrm{mg} / \mathrm{L}, \mathrm{NO} 3$ : Nitrate in $\mathrm{mg} / \mathrm{L}, \mathrm{SO} 4$ : Sulphate in $\mathrm{mg} / \mathrm{L}, \mathrm{Cl}$ : Chloride in mg/L, Max: Maximum value, Min: Minimum value, SD: Standard deviation, CV: Coefficient of variation

Table 2: Characteristics of Lake water at sampling point 2

\begin{tabular}{|c|c|c|c|c|c|c|c|c|c|c|c|c|c|}
\hline \multirow{2}{*}{ Date } & \multicolumn{2}{|c|}{\begin{tabular}{|l|} 
Temperature \\
\end{tabular}} & \multirow{2}{*}{$\mathrm{pH}$} & \multirow{2}{*}{ Alkalinity } & \multirow{2}{*}{ Do } & \multirow{2}{*}{$B O D$} & \multirow{2}{*}{ TDS } & \multirow{2}{*}{ TH } & \multirow{2}{*}{ Ca } & \multirow{2}{*}{$\mathrm{Mg}$} & \multirow{2}{*}{$\mathrm{NO}_{3}$} & \multirow{2}{*}{$\mathrm{SO}_{4}$} & \multirow{2}{*}{ cl } \\
\hline & atms & water & & & & & & & & & & & \\
\hline 5-Nov & 30.2 & 24.4 & 7.96 & 7.39 & \begin{tabular}{|l|l|}
8.28 \\
\end{tabular} & 96.39 & 308 & 170.46 & 127.85 & 12.62 & 0.92 & 3.56 & 211.5 \\
\hline 8-Nov & 29.71 & 24.05 & 7.92 & 04 & 8.37 & 97.1 & 306 & 166.73 & 125.04 & 41.68 & .89 & 3.58 & 209.38 \\
\hline 11-Nov & 29.22 & 23.7 & 7.85 & & 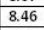 & 81 & & 163 & \begin{tabular}{|l|l|}
122.25 \\
\end{tabular} & 40.75 & .87 & 61 & 7.24 \\
\hline 14-Nov & 28.73 & 23.35 & 8.01 & & 8.28 & 9857 & & 159.26 & 119.44 & 39.81 & 8.86 & 3.62 & 205.11 \\
\hline & & & & & & & & & & & & & \\
\hline $20-n$ & & 22.65 & $\%$ & & & & & & & & & & 84 \\
\hline 23-Nov & 26 & 21.3 & 7.94 & & 8.28 & & 299 & & & $\begin{array}{ll} & \\
37.01\end{array}$ & 1.84 & 66 & \\
\hline $26-\mathrm{N}$ & .77 & 21.95 & 7.96 & & 8.2 & 10 & & 27 & \begin{tabular}{|l|}
108.2 \\
\end{tabular} & $\begin{array}{lll}36.07 & \\
\end{array}$ & .83 & 68 & 96.31 \\
\hline $29-1$ & & $m$ & 7.85 & & & & & & & & & & \\
\hline & & & & & & & & & & & & & \\
\hline 5-De & 25.46 & 21.3 & 7.9 & & & & 29 & 140.92 & & 35.23 & 79 & & 33.39 \\
\hline 8-De & 25.12 & 21. & 8.05 & & 7.6 & 10 & 29 & 14 & & 36.25 & 78 & 38 & 94.54 \\
\hline $11-0$ & 24 & 21 & 7.9 & & & & & & & & & & 7.7 \\
\hline & & & 7. & & & & & & & & & & 6.85 \\
\hline $17-5$ & & & 7.7 & & & & & & & & & & \\
\hline $20-5$ & & & 7.8 & & & & & & & & & & 05 \\
\hline $23-5$ & & 21.6 & 7. & & 6.9 & 107 & 30 & & 1 & 15 & 74 & 88 & 200.3 \\
\hline $26-0$ & & & & & & & & & & & & & \\
\hline $\mathrm{Ma}$ & 2 & 24.4 & 8. & & 8.46 & 10 & 308 & & & 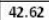 & 92 & 69 & 11.5 \\
\hline M & & & & & & & & & & & & & \\
\hline $\mathrm{Me}$ & & & & & & 102 & 301 & & & & & & \\
\hline 5.5 & 2. & 1.0 & 0.0 & & 0. & & 4.5 & & & 2.6 & 0.0 & 0.29 & \\
\hline , & 0.088 & \begin{tabular}{|l|}
0.047 \\
\end{tabular} & 0.01 & 067 & 0.074 & 0.036 & 0.015 & 0.067 & 0.06 & 0.067 & 0.06 & 0.085 & 0.02 \\
\hline
\end{tabular}

Table 3: Characteristics of Lake water at sampling point 3

\begin{tabular}{|c|c|c|c|c|c|c|c|c|c|c|c|c|c|}
\hline & \multicolumn{2}{|c|}{ Temperature } & $\mathrm{pH}$ & Alkalinity & Do & BOD & TDS & TH & $c a$ & $\mathrm{Mg}$ & $\mathrm{NO}_{3}$ & $\mathrm{SO}_{4}$ & d \\
\hline & \begin{tabular}{|l} 
atms \\
302
\end{tabular} & water & 778 & 105 & 81 & 9425 & 301 & 6.67 & 125 & 4167 & 00 & 348 & 2068 \\
\hline 8-Nov & 29.71 & 24 & 7.74 & & 8.18 & 94.25 & 299 & & 122.27 & 40.76 & 0.87 & 3.5 & 04.72 \\
\hline vo & 29.22 & 23.7 & 67 & & 27 & & & & 119.53 & 39.84 & 0.85 & 53 & 22.64 \\
\hline Nov & 2 & 23.35 & 7.83 & & 8.1 & 96.33 & 297 & & 116.79 & 38.93 & 84 & 54 & 55 \\
\hline N & 24 & & 7.79 & & 01 & 970 & & 2.06 & 114.05 & 38.02 & 08 & 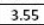 & 98.48 \\
\hline & 75 & 22.6 & & & & & & & 111. & & & & \\
\hline & 6 & 21 & 7. & & & & & & & & & & \\
\hline $26-1$ & & 21.9 & 7.7 & & & & & & 10 & & & & 91.95 \\
\hline 29-Nov & 28 & 21.6 & & & 18 & & & & 103.17 & 34.39 & & & \\
\hline 2-Dec & 79 & 21.25 & 7.66 & & 74 & 10 & & & 100 & 33.46 & 0.7 & 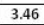 & .97 \\
\hline $5-\mathrm{D}$ & & 21.3 & 7. & & 41 & & & & & & & & 09 \\
\hline & & & & & & & & & & & & & \\
\hline & & & & & 7.25 & & & & & & & & \\
\hline 14-Ded & 1.47 & 21.44 & 7.6 & & & 13 & & 149 & 112 & & 0. & & 192 \\
\hline $7-0$ & & 21.5 & 7.57 & & .76 & & & & & & 0. & & \\
\hline $20-5$ & 23 & 21.58 & 7.67 & & & & & & & & & & \\
\hline & & & 7.78 & & & & & & & & & & \\
\hline & & & 7.5 & & & & & & & & & & \\
\hline $\mathrm{M}^{2}$ & $\frac{22 .}{30 .}$ & \begin{tabular}{|l}
24.0 \\
24. \\
\end{tabular} & 7.87 & & & 106.03 & & 56.67 & 12 & $\begin{array}{l}41.42 \\
41.67\end{array}$ & $\begin{array}{l}0.11 \\
0.9 \\
\end{array}$ & 61 & 206.8 \\
\hline & 22.82 & & & & & & & & 100. & & & & \\
\hline $\mathrm{M}$ & & & & & & & & & & & & & \\
\hline 5.5 & & & & & & & & & & & & & \\
\hline$c . V$ & & & & & & 0.036 & 0.014 & 0.007 & 0.007 & 0.067 & 0.063 & 0.084 & 0.029 \\
\hline
\end{tabular}

Table 4: Characteristics of Lake water at sampling point 4

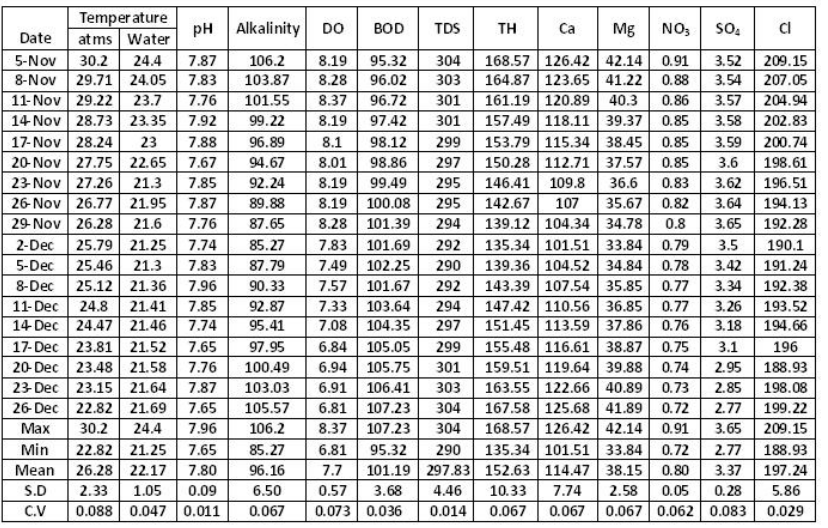

Table 5: Characteristics of Lake water at sampling point 5

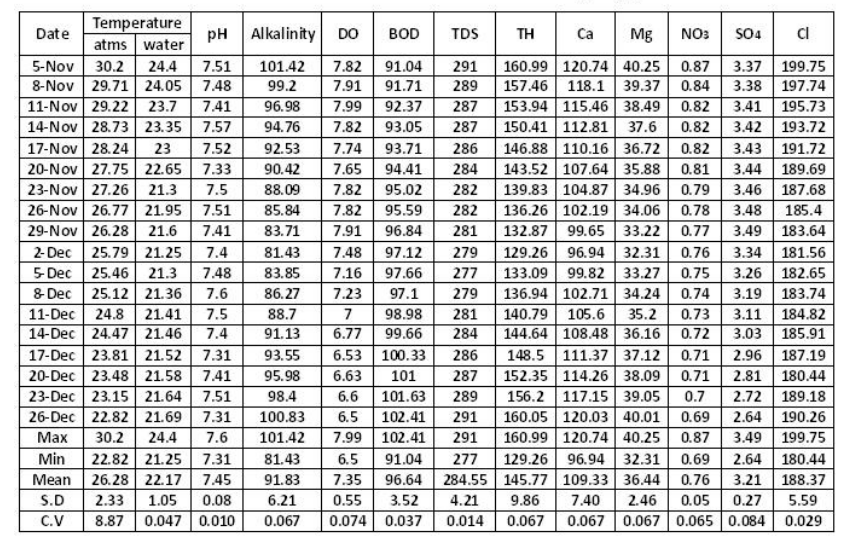


Table 6: Correlation Co-efficient of different parameters at sampling point 1

\begin{tabular}{|c|c|c|c|c|c|c|c|c|c|c|c|}
\hline & $\mathrm{pH}$ & Alk & $\mathrm{DO}$ & $\mathrm{BOD}$ & $\mathrm{TDS}$ & $\mathrm{TH}$ & $\mathrm{Ca}$ & $\mathrm{Mg}$ & $\mathrm{NO}_{3}$ & $\mathrm{SO}_{4}$ & $\mathrm{~d}$ \\
\hline $\mathrm{pH}$ & - & - & - & - & - & - & - & - & - & - & - \\
\hline $\mathrm{Allk}$ & -0.060 & - & - & - & - & - & - & - & - & - & - \\
\hline $\mathrm{DO}$ & 0.179 & -0.181 & - & - & - & - & - & - & - & - & - \\
\hline $\mathrm{BOD}$ & -0.216 & -0.072 & -0.919 & - & - & - & - & - & - & - & - \\
\hline $\mathrm{TDS}$ & -0.129 & 0.966 & -0.102 & -0.099 & - & - & - & - & - & - & - \\
\hline $\mathrm{TH}$ & -0.060 & 0.485 & -0.181 & -0.072 & 0.966 & - & - & - & - & - & - \\
\hline $\mathrm{Ca}$ & -0.060 & 0.485 & -0.182 & -0.072 & 0.966 & 0.485 & - & - & - & - & - \\
\hline $\mathrm{Mg}$ & -0.060 & 0.485 & -0.181 & -0.072 & 0.966 & 0.485 & 0.485 & - & - & - & - \\
\hline $\mathrm{NO}_{3}$ & 0.179 & 0.144 & 0.897 & -0.990 & 0.182 & 0.144 & 0.144 & 0.144 & - & - & - \\
\hline $\mathrm{SO}_{4}$ & 0.171 & -0.418 & 0.943 & -0.857 & -0.349 & -0.418 & -0.148 & -0.418 & 0.824 & - & - \\
\hline $\mathrm{Cl}$ & 0.001 & 0.733 & 0.440 & -0.659 & 0.727 & 0.733 & 0.733 & 0.733 & 0.706 & 0.240 & - \\
\hline
\end{tabular}

Table 7: Correlation Co-efficient of different parameters at sampling point 2

\begin{tabular}{|c|c|c|c|c|c|c|c|c|c|c|c|}
\hline & $\mathrm{pH}$ & Alk & $\mathrm{DO}$ & $\mathrm{BOD}$ & $\mathrm{TOS}$ & $\mathrm{TH}$ & $\mathrm{Ca}$ & $\mathrm{Mg}$ & $\mathrm{NO}_{3}$ & $\mathrm{SO}_{4}$ & $\mathrm{Cl}$ \\
\hline $\mathrm{pH}$ & - & - & - & - & - & - & - & - & - & - & - \\
\hline $\mathrm{Alk}$ & -0.097 & - & - & - & - & - & - & - & - & - & - \\
\hline $\mathrm{DO}$ & 0.370 & -0.181 & - & - & - & - & - & - & - & - & - \\
\hline $\mathrm{BOD}$ & -0.392 & -0.072 & -0.919 & - & - & - & - & - & - & - & - \\
\hline $\mathrm{TDS}$ & -0.161 & 0.962 & -0.097 & -0.099 & - & - & - & - & - & - & - \\
\hline $\mathrm{TH}$ & -0.097 & 0.485 & -0.181 & -0.072 & 0.962 & - & - & - & - & - & - \\
\hline $\mathrm{Ca}$ & -0.097 & 0.485 & -0.182 & -0.072 & 0.962 & 0.485 & - & - & - & - & - \\
\hline $\mathrm{Mg}$ & -0.097 & 0.485 & -0.182 & -0.071 & 0.962 & 0.485 & 0.485 & - & - & - & - \\
\hline $\mathrm{NO}_{3}$ & 0.307 & 0.153 & 0.889 & -0.984 & 0.200 & 0.153 & 0.153 & 0.153 & - & - & - \\
\hline $\mathrm{SO}_{4}$ & 0.327 & -0.421 & 0.942 & -0.856 & -0.346 & -0.421 & -0.421 & -0.421 & 0.817 & - & - \\
\hline $\mathrm{Cl}^{\mathrm{C}}$ & 0.138 & 0.733 & 0.440 & -0.659 & 0.727 & 0.733 & 0.733 & 0.733 & 0.708 & 0.241 & - \\
\hline
\end{tabular}

Table 8: Correlation Co-efficient of different parameters at sampling point 3

\begin{tabular}{|c|c|c|c|c|c|c|c|c|c|c|c|}
\hline & $\mathrm{pH}$ & $\mathrm{Alk}$ & $\mathrm{DO}$ & $\mathrm{BOD}$ & $\mathrm{TDS}$ & $\mathrm{TH}$ & $\mathrm{Ca}$ & $\mathrm{Mg}$ & $\mathrm{NO}_{3}$ & $\mathrm{SO}_{4}$ & $\mathrm{Cl}$ \\
\hline $\mathrm{pH}$ & - & - & - & - & - & - & - & - & - & - & - \\
\hline Alk & -0.103 & - & - & - & - & - & - & - & - & - & - \\
\hline $\mathrm{DO}$ & 0.366 & -0.181 & - & - & - & - & - & - & - & - & - \\
\hline $\mathrm{BOD}$ & -0.388 & -0.071 & -0.920 & - & - & - & - & - & - & - & - \\
\hline $\mathrm{TDS}$ & -0.167 & 0.965 & -0.117 & -0.093 & - & - & - & - & - & - & - \\
\hline $\mathrm{TH}$ & -0.103 & 0.485 & -0.181 & -0.071 & 0.965 & - & - & - & - & - & - \\
\hline $\mathrm{Ca}$ & -0.103 & 0.485 & -0.181 & -0.072 & 0.965 & 0.485 & - & - & - & - & - \\
\hline $\mathrm{Mg}$ & -0.103 & 0.485 & -0.180 & -0.072 & 0.965 & 0.485 & 0.485 & - & - & - & - \\
\hline $\mathrm{NO}_{3}$ & 0.330 & 0.140 & 0.892 & -0.989 & 0.170 & 0.140 & 0.140 & 0.141 & - & - & - \\
\hline $\mathrm{SO}_{4}$ & 0.327 & -0.420 & 0.944 & -0.856 & -0.358 & -0.420 & -0.420 & -0.420 & 0.822 & - & - \\
\hline $\mathrm{d}$ & 0.135 & 0.733 & 0.441 & -0.659 & 0.729 & 0.733 & 0.733 & 0.733 & 0.701 & 0.243 & - \\
\hline
\end{tabular}

Table 9: Correlation Co-efficient of different parameters at sampling point 4

\begin{tabular}{|c|c|c|c|c|c|c|c|c|c|c|c|}
\hline & $\mathrm{pH}$ & Alk & $\mathrm{DO}$ & $\mathrm{BOD}$ & $\mathrm{TDS}$ & $\mathrm{TH}$ & $\mathrm{Ca}$ & $\mathrm{Mg}$ & $\mathrm{NO}_{3}$ & $\mathrm{SO}_{4}$ & $\mathrm{Cl}$ \\
\hline $\mathrm{pH}$ & - & - & - & - & - & - & - & - & - & - & - \\
\hline Alk & -0.097 & - & - & - & - & - & - & - & - & - & - \\
\hline $\mathrm{DO}$ & 0.371 & -0.179 & - & - & - & - & - & - & - & - & - \\
\hline $\mathrm{BOD}$ & -0.392 & -0.072 & -0.919 & - & - & - & - & - & - & - & - \\
\hline $\mathrm{TDS}$ & -0.140 & 0.986 & -0.103 & -0.094 & - & - & - & - & - & - & - \\
\hline $\mathrm{TH}$ & -0.097 & 0.485 & -0.180 & -0.072 & 0.967 & - & - & - & - & - & - \\
\hline $\mathrm{Ca}$ & -0.097 & 0.485 & -0.180 & -0.072 & 0.967 & 0.485 & - & - & - & - & - \\
\hline $\mathrm{Mg}$ & -0.097 & 0.485 & -0.179 & -0.072 & 0.968 & 0.485 & 0.485 & - & - & - & - \\
\hline $\mathrm{NO}_{3}$ & 0.135 & 0.149 & 0.889 & -0.984 & 0.179 & 0.149 & 0.149 & 0.149 & - & - & - \\
\hline $\mathrm{SO}_{4}$ & 0.329 & -0.421 & 0.941 & -0.855 & -0.359 & -0.422 & -0.422 & -0.422 & 0.818 & - & - \\
\hline $\mathrm{d}$ & 0.138 & 0.733 & 0.441 & -0.659 & 0.723 & 0.733 & 0.733 & 0.733 & 0.706 & 0.240 & - \\
\hline
\end{tabular}

Table 10: Correlation Co-efficient of different parameters at sampling point 5

\begin{tabular}{|c|c|c|c|c|c|c|c|c|c|c|c|}
\hline & $\mathrm{pH}$ & Alk & $\mathrm{DO}$ & $\mathrm{BOD}$ & $\mathrm{TDS}$ & $\mathrm{TH}$ & $\mathrm{Ca}$ & $\mathrm{Mg}$ & $\mathrm{NO}_{3}$ & $\mathrm{SO}_{4}$ & $\mathrm{Cl}$ \\
\hline $\mathrm{pH}$ & - & - & - & - & - & - & - & - & - & - & - \\
\hline Alk & -0.112 & - & - & - & - & - & - & - & & - & - \\
\hline $\mathrm{DO}$ & 0.371 & -0.182 & - & - & - & - & - & - & - & - & - \\
\hline $\mathrm{BOD}$ & -0.392 & -0.072 & -0.919 & - & - & - & - & - & - & - & - \\
\hline $\mathrm{TDS}$ & -0.184 & 0.965 & -0.116 & -0.086 & - & - & - & - & - & - & - \\
\hline $\mathrm{TH}$ & -0.112 & 0.485 & -0.182 & -0.072 & 0.965 & - & - & - & - & - & - \\
\hline $\mathrm{Ca}$ & -0.112 & 0.485 & -0.182 & -0.072 & 0.965 & 0.485 & - & - & - & - & - \\
\hline $\mathrm{Mg}$ & -0.112 & 0.485 & -0.182 & -0.072 & 0.965 & 0.485 & 0.485 & - & - & - & - \\
\hline $\mathrm{NO}_{3}$ & 0.340 & 0.139 & 0.898 & -0.986 & 0.172 & 0.139 & 0.139 & 0.139 & - & - & - \\
\hline $\mathrm{SO}_{4}$ & 0.336 & -0.417 & 0.944 & -0.857 & -0.356 & -0.417 & -0.417 & -0.417 & 0.823 & - & - \\
\hline $\mathrm{Cl}$ & 0.130 & 0.733 & 0.439 & -0.659 & 0.727 & 0.733 & 0.733 & 0.733 & 0.695 & 0.245 & - \\
\hline
\end{tabular}

Correlation Coefficients (r): In all the sampling points, the highest positive correlation is found between Total dissolved solids \& Alkalinity (TDS \& $\mathrm{HCO}_{3}$ ), Chloride \& Alkalinity $\left(\mathrm{Cl} \& \mathrm{HCO}_{3}\right)$, Nitrate \& Dissolved Oxygen $\left(\mathrm{NO}_{3} \& \mathrm{DO}\right)$, Sulphate \& Dissolved Oxygen $\left(\mathrm{SO}_{4} \& \mathrm{DO}\right)$, Total hardness \& Total dissolved solids (TH \& TDS), Magnesium hardness \& Total dissolved solids (Mg \& TDS), Chloride \& Total dissolved solids ( $\mathrm{Cl} \& \mathrm{TDS})$, Chloride \& Total hardness $(\mathrm{Cl} \&$ $\mathrm{TH})$, Calcium hardness \& Chloride $(\mathrm{Ca} \& \mathrm{Cl})$, Magnesium hardness \& Chloride $(\mathrm{Mg} \& \mathrm{Cl})$, Nitrate \& Chloride $\left(\mathrm{NO}_{3} \&\right.$ $\mathrm{Cl})$, Nitrate \& Sulphate $\left(\mathrm{NO}_{3} \& \mathrm{SO}_{4}\right)$.

Factor Analysis: In all the sampling points, almost following factor 1 and factor2 gave the loadings.

\section{Factor 1:}

(1) Strong loading: It suggests that the quality of ground water is mainly controlled by strong loading parameters. The Strong loading on $\mathrm{TH}, \mathrm{Mg} \& \mathrm{Ca}$ shows the permanent hardness of water. Alkalinity, $\mathrm{Cl}$ also suggests the hardness of water. High TDS shows that wash water from the water treatment are being added up in the fresh water of lake.

(2) Moderate loading: No loading at all.

(3) Weak loading: No loading at all.

\section{Factor 2:}

(1) Strong loading: Strong loading on DO suggests that water is being aerated in water treatment units of Gulbarga. $\mathrm{NO}_{3}, \&$ $\mathrm{SO}_{4}$ shows mild pollution and inherited quality of river water

(2) Moderate loading: Loading of $\mathrm{Cl}$ also indicates pollution \& permanent hardness of water.

(3) Weak loading: No loading at all. 
Table 11: Factor Analysis (Sample 1) (Rotated Factor Loadings and Communalities, Varimax Rotation)

\begin{tabular}{|c|c|c|c|}
\hline Variable & Factor 1 & Factor 2 & Communality \\
\hline $\mathrm{pH}$ & -0.076 & 0.246 & 0.066 \\
\hline Alkalinity & 0.994 & -0.091 & 0.997 \\
\hline DO & -0.092 & 0.970 & 0.950 \\
\hline $\mathrm{BOD}$ & -0.160 & -0.979 & 0.984 \\
\hline $\mathrm{TDS}$ & 0.974 & -0.044 & 0.950 \\
\hline $\mathrm{TH}$ & 0.994 & -0.091 & 0.997 \\
\hline $\mathrm{Ca}$ & 0.994 & -0.091 & 0.997 \\
\hline $\mathrm{Mg}$ & 0.994 & -0.091 & 0.996 \\
\hline $\mathrm{NO} 3$ & 0.234 & 0.963 & 0.982 \\
\hline SO4 & -0.332 & 0.934 & 0.982 \\
\hline $\mathrm{Cl}$ & 0.797 & 0.545 & 0.982 \\
\hline Eigen value & 5.8 & 4.0 & - \\
\hline \% Variance & 52.2 & 37.2 & - \\
\hline $\begin{array}{c}\text { Cumulative } \% \\
\text { of Variance }\end{array}$ & 52.2 & 89.4 & - \\
\hline
\end{tabular}

T able 12: Factor Analysis (Sample 2) (Rotated Factor Loadings and Communalities, Varimax Rotation)

\begin{tabular}{|c|c|c|c|}
\hline Variable & Factor 1 & Factor 2 & Communality \\
\hline $\mathrm{pH}$ & -0.095 & 0.345 & 0.214 \\
\hline Alkalinity & 0.995 & -0.081 & 0.996 \\
\hline $\mathrm{DO}$ & -0.101 & 0.967 & 0.945 \\
\hline $\mathrm{BOD}$ & -0.15 & -0.98 & 0.983 \\
\hline $\mathrm{TDS}$ & 0.972 & -0.033 & 0.945 \\
\hline $\mathrm{TH}$ & 0.995 & -0.081 & 0.996 \\
\hline $\mathrm{Ca}$ & 0.995 & -0.081 & 0.996 \\
\hline $\mathrm{Mg}$ & 0.995 & -0.082 & 0.996 \\
\hline NO3 & 0.235 & 0.955 & 0.967 \\
\hline SO4 & -0.342 & 0.925 & 0.972 \\
\hline $\mathrm{Cl}$ & 0.791 & 0.554 & 0.932 \\
\hline Eigen value & 5.8 & 4.0 & - \\
\hline \% Variance & 52.2 & 37.2 & - \\
\hline $\begin{array}{c}\text { Cumulative } \% \\
\text { of Variance }\end{array}$ & 52.2 & 89.4 & - \\
\hline
\end{tabular}

Table 13: Factor Analysis (Sample 3) (Rotated Factor Loadings and Communalities, Varimax Rotation)

\begin{tabular}{|c|c|c|c|}
\hline Variable & Factor 1 & Factor 2 & Communality \\
\hline $\mathrm{pH}$ & -0.099 & 0.345 & 0.218 \\
\hline Alkalinity & 0.995 & -0.082 & 0.996 \\
\hline DO & -0.101 & 0.966 & 0.943 \\
\hline BOD & -0.152 & -0.98 & 0.983 \\
\hline TDS & 0.974 & -0.047 & 0.951 \\
\hline TH & 0.995 & -0.082 & 0.996 \\
\hline $\mathrm{Ca}$ & 0.995 & -0.081 & 0.996 \\
\hline Mg & 0.995 & -0.081 & 0.996 \\
\hline $\mathrm{NO}_{3}$ & 0.222 & 0.96 & 0.97 \\
\hline $\mathrm{SO}_{4}$ & -0.341 & 0.924 & 0.97 \\
\hline $\mathrm{Cl}$ & 0.792 & 0.554 & 0.935 \\
\hline Eigen value & 5.8 & 4.2 & - \\
\hline$\%$ Variance & 52.2 & 37.2 & - \\
\hline $\begin{array}{c}\text { Cumulative } \% \\
\text { of Variance }\end{array}$ & 52.2 & 89.4 & - \\
\hline
\end{tabular}

Table 14: Factor Analysis (Sample 4) (Rotated Factor Loadings and Communalities, Varimax Rotation)

\begin{tabular}{|c|c|c|c|}
\hline Variable & Factor 1 & Factor 2 & Communality \\
\hline $\mathrm{pH}$ & -0.091 & 0.456 & 0.216 \\
\hline Alkalinity & 0.995 & -0.079 & 0.997 \\
\hline DO & -0.101 & 0.966 & 0.943 \\
\hline BOD & -0.149 & -0.98 & 0.982 \\
\hline TDS & 0.974 & -0.039 & 0.951 \\
\hline TH & 0.995 & -0.079 & 0.997 \\
\hline $\mathrm{Ca}$ & 0.995 & -0.079 & 0.997 \\
\hline Mg & 0.995 & -0.079 & 0.997 \\
\hline $\mathrm{NO}_{3}$ & 0.227 & 0.956 & 0.966 \\
\hline $\mathrm{SO}_{4}$ & -0.346 & 0.923 & 0.972 \\
\hline $\mathrm{Cl}$ & 0.789 & 0.555 & 0.932 \\
\hline Eigen value & 5.7 & 4.1 & - \\
\hline$\%$ Variance & 52.2 & 38.2 & - \\
\hline $\begin{array}{c}\text { Cumulative } \% \\
\text { of variance }\end{array}$ & 52.2 & 89.4 & - \\
\hline
\end{tabular}


Table 15: Factor Analysis (Sample 5) (Rotated Factor Loadings and Communalities, Varimax Rotation)

\begin{tabular}{|c|c|c|c|}
\hline Variable & Factor 1 & Factor 2 & Communality \\
\hline $\mathrm{pH}$ & -0.11 & 0.349 & 0.227 \\
\hline Alkalinity & 0.994 & -0.083 & 0.996 \\
\hline DO & -0.099 & 0.967 & 0.944 \\
\hline BOD & -0.153 & -0.979 & 0.982 \\
\hline TDS & 0.974 & -0.051 & 0.951 \\
\hline TH & 0.994 & -0.083 & 0.996 \\
\hline $\mathrm{Ca}$ & 0.994 & -0.083 & 0.996 \\
\hline $\mathrm{Mg}$ & 0.994 & -0.083 & 0.996 \\
\hline $\mathrm{NO}_{3}$ & 0.222 & 0.960 & 0.971 \\
\hline $\mathrm{SO}_{4}$ & -0.336 & 0.925 & 0.968 \\
\hline $\mathrm{Cl}$ & 0.973 & 0.551 & 0.933 \\
\hline Eigen value & 5.9 & 4.3 & - \\
\hline$\%$ Variance & 52.2 & 38.2 & - \\
\hline $\begin{array}{c}\text { Cumulative } \% \\
\text { of variance }\end{array}$ & 52.2 & 89.4 & - \\
\hline
\end{tabular}

\section{CONCLUSIONS}

The Physico-chemical characteristics are high at North-West corner (S1) where as South-East corner (S2), Centre (S3), and North-East corner (S4) are moderate \& the South-West corner (S5) are low. Since the lake is receiving fresh cum treated water from Bhima River, the water quality is good. All anthropogenic activities are barred in the lake water viz., bathing, swimming, Cattle's washing etc., since the lake is well protected by pitching and fencing. The Physico-chemical Characteristics of Shri Sharanabasaveshwara lake at different sampling points were analyzed and found all the values within the permissible limits except BOD which may be because of wash water from treatment unit. The $\mathrm{pH}$ in Shri Sharanabasaveshwara lake is in between 7.45 to 8.77 . The DO in Shri Sharanabasaveshwara lake varies from 7.35 to 8.65 $\mathrm{mg} / \mathrm{L}$. The BOD in Shri Sharanabasaveshwara lake varies from 96.65 to $113.70 \mathrm{mg} / \mathrm{L}$. Factor analysis at all the sampling points has shown that $\mathrm{DO}$ is rich in water, NO3 and SO4 are present in the water is an inherent quality of Bhima river water. Factor analysis for sampling points shown moderate high value in chloride, which shows permanent hardness of water. In all the sampling points, the highest positive correlation is found between Chloride \& Alkalinity $(\mathrm{Cl} \&$ $\mathrm{HCO} 3)$, Chloride \& Total hardness ( $\mathrm{Cl} \& \mathrm{TH})$, Calcium hardness \& Chloride $(\mathrm{Ca} \& \mathrm{Cl})$, Magnesium hardness \& Chloride $(\mathrm{Mg} \& \mathrm{Cl})$, Nitrate \& Chloride (NO3 \& $\mathrm{Cl}$ ), Nitrate $\&$ Sulphate (NO3 \& SO4) this shows the permanent hardness of water. Shri Sharanabasaveshwara lake is now being utilized only for boating, even fishing activities as been stopped by authorities.

\section{ACKNOWLEDGEMENTS}

The authors are thankful to the "Hyderabad Karnataka Education Society Gulbarga" Karnataka-State, India.

\section{REFERENCES}

[1] Wood, R.B. and G.E. Gibson, Eutrophication and Loch Heagen. Water Res. 1974, 7, 163-287.

[2] Hemasundaram, A. K. Dhanalakshmi, B. Prasad and N.V.S. Naidu, Assessment of water quality with regard to surfactants in pilgrim town - a case study of Tirupati. Ultr. Scient. Phyl. Sci. 2003, 15(2): 189-194.

[3] Kajak, Z. Eutrofizacja jezior (Eutrophication of lakes), Warszawa, PWN, 1979, 233 p.

[4] Golterman.H.L and N.T. Qude, Eutrophication of lakes, rivers and coastal seas, The handbook of environmental chemistry (Ed. O.Hutzinger), Berlin Heidelberg, SpringerVerlag, 1991, 5(A): 70-124.

[5] Freeman.C., M.A. Lock and B. Reynolds, Climatic change and the release of immobilized nutrients from Welsh riparian wetland soils, Ecol. Eng., 1993, 2(4): 367- 373.

[6] Clair T.A., B.G. Sayer, J.R. Kramer and D.R.Eaton, Seasonal variation in the composition of aquatic organic matter in some Nova Scotion brown waters: A nuclear resonance approach, Hydrobiologia, 1996, 317(2): 141- 150.

[7] Dortch M.S., Removal of solids, nitrogen and phosphorus in the Cache River wetland, Wetlands, 1996, 16(3): 358-365.

[8] Craft C.B., 1997, Dynamics of nitrogen and phosphorus retention during wetland ecosystem succession, Wetland Ecology Management, 1997, 4(3): 177-187.

[9] Ansari A.H and S. Kumar, Effect of nitrate input on the release of phosphorus from a tropical lake sediment, Dimens. Polln., 2003, 2:120-126.

[10] Singh K.P., A. Malik, D. Mohan and S. Sinha, "Multivariate Statistical Techniques for the Evaluation of Spatial and Temporal Variations in Water Quality of Gomti River (India) - a Case Study”, Water Research, 2004, 38 (18), PP 3980-3992.

[11] Kowalkowski T., R. Zbytniewski, J. Szpejna and B. Buszewski, Application of Chemo metrics in River Water Classification, Journal of Water Research, 2006, 40(4), PP. 744-752.

[12] Boyacioglu. H. and H. Boyacioglu, "Water Pollution Sources Assessment by Multivariate Statistical Methods in the Tahtali Basin, Turkey", Journal of Environmental Geology, 2006, 54(2), PP. 275-282.

[13] Liu. C.W., Lin K.H. and Kuo Y.M., Application of factor analysis in the assessment of groundwater quality in a black foot disease area in Taiwan, The Science of Total Environment, 2003, Vol. 313, pp77-89. 


\section{BIOGRAPHIES}

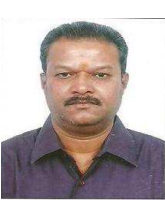

Shivasharanappa, Teaching M. Tech (Environmental -Engineering) since from 20 years, published 11 papers in international journals, 2 in national conferences \& 1 in international conference.

Bettad Prakash, B.E (Civil) in First Class, M. Tech (Environmental Engineering) IVth sem student, P D A College of Engineering Gulbarga, Karnataka 